E.L.U.A., 6, 1990, pp: 183-206.

\title{
VARIACIONISMO Y ADQUISICIÓN DE UNA SEGUNDA LENGUA: ESTUDIO DE UNA COMUNIDAD FRANCÓFONA
}

\author{
MARÍA ROSARIO HERNÁNDEZ \\ (Universidad de Alicante)
}

\section{Introducción}

Cualquier investigador que se interese por la ciencia del lenguaje y que pretenda hoy analizar la lengua, tanto sincrónica como históricamente, si parte de unconcepto dinámico de la misma, y la considera como proceso — no como producto-, y como una forma de comportamiento social, no puede prescindir ni del usuario de la lengua ni del contexto social en que ésta es usada. Consecuentemente, la perspectiva más adecuada de enfocar su estudio sería la sociolinguística.

$\mathrm{Si}$, por otra parte, este supuesto investigador admite la existencia ya innegable de la variación lingüística, cuyo carácter sistemático e inherente ha sido ampliamente reconocido desde el trabajo significativo de Labov (1966), su perspectiva sociolingüística puede encontrar en la corriente variacionista una dirección acertada.

Después de numerosos trabajos, la sociolingüística puede ofrecer hoy, para los estudios de la variación, una metodología y un modelo teórico claramente definidos que permiten traducir a coeficientes de probabilidad la covariación de unas variables lingüísticas con unos factores lingüísticos y sociales. De este modo, supera el simple aspecto descriptivo de la lengua para explicar la competencia sociolingüística de las comunidades de habla mediante un modelo de regla variable (vid. Rousseau y Sankoff, 1978).

Es evidente que la corriente variacionista encuentra su aplicación en una gran diversidad de situaciones lingüísticas dentro de comunidades, bien monolingües bien multilingües. Prueba de ello son los numerosos estudios publicados hasta la fecha sobre variedades diafásicas o diastráticas, principalmente en zonas urbanas, y sobre los múltiples temas derivados del multilingüismo, sin olvidar otros campos como la pragmática o la etnografía de la comunicación. 
Las comunidades multilingües — por ser actualmente la regla más que la excepción- - están siendo objeto de un constante y creciente interés por parte de lingüistas, sociólogos, etnólogos y pedagogos deseosos de conectar sus respectivas parcelas de investigación con la variación lingüística.

Una de estas parcelas directamente conectada con los estudios sobre multilingüismo nos resulta especialmente atractiva. Nos referimos a la investigación centrada en la adquisición de una segunda lengua. Nuestro interés se explica por la complejidad del tema y por los problemas, muchos de ellos aún sin resolver, que plantea a varias disciplinas: lingüística, psicolingüística, pragmática, entre otras. Esta área de investigación ha sido examinada durante largo tiempo por caminos separados, sin visión integradora. Muchas veces se ha considerado bajo su aspecto didáctico o psicolingüístico, sin tener en cuenta los últimos avances de las teorías linguísticas y más concretamente de los recientes estudios sociolingüísticos.

Un modesto intento de acercamiento al referido tema, mediante el enfoque variacionista de la sociolingüística laboviana, lo constituye nuestro estudio empírico sobre un grupo de adolescentes franceses del Lycée Français de Alicante, realizado no hace mucho tiempo.

Pero antes de entrar en el desarrollo de dicha investigación, cabe plantear unas previas consideraciones centradas en el aspecto teórico y metodológico sobre las parcelas que intentamos relacionar: por una parte, el análisis de la variación lingüística desde una perspectiva sociolingüística; por otra, el proceso de adquisición de una segunda lengua. Un marco más general, el de los universales del lenguaje, puede constituir una fuente útil para la formulación de unos principios básicos sobre los cuales vayan articuladas las dos áreas antes citadas.

\section{Las propuestas universalistas}

Uno de los aciertos de la linguística generativa y particularmente de las propuestas chomskyanas es de haber ofrecido una teoría de la facultad humana del lenguaje, una teoría explicativa para el estudio de lo que constituye el conocimiento de una lengua, es decir, el sistema de reglas internalizado por un hablante-oyente ideal. La naturaleza de este sistema se define a partir de una hipótesis formulada según unos principios válidos para todas las lenguas, los cuales determinan la forma de la gramática. Estos principios constituyen lo que tradicionalmente - al menos desde mediados del siglo XVII, con la linguística cartesiana - se ha denominado «gramática universal» (GU), es decir, una gramática que es expresión de las propiedades biológicamente necesarias del lenguaje humano.

Uno de los objetivos de esta GU es la formulación de unos parámetros que dan cuenta de unos principios universales innatos a partir de los cuales el niño va configurando su gramática durante su proceso de adquisición del lenguaje, ayudado por la entrada de datos que están a su alcance. Estos parámetros permiten explicar la escasa variación que existe entre las lenguas y la relativa rapidez y uniformidad que caracterizan la adquisición del lenguaje (vid. Chomsky, 1965; 1971). 
De este modo, la gramática de una lengua particular sigue los principios de la GU, pero con una articulación adicional. Cuando el niño adquiere su primera lengua, esta GU interactúa entre la entrada lingüística y la gramática particular de la lengua en cuestión.

2.1. Entre los estudios centrados en el conjunto de las lenguas naturales, podemos distinguir los que intentan clasificar las lenguas desde un aspecto diacrónico, en función de su genealogía o de su distribución geográfica, de aquellos otros - llamados tipológicos - que, desde una perspectiva sincrónica, se interesan por los diferentes tipos de lenguas a partir del isomorfismo de su estructura, y proponen dos sistemas, uno basado en criterios morfológicos, otro en criterios sintácticos.

Entre estos últimos, podemos encontrar una alternativa a la GU para abordar el problema de los universales del lenguaje, principalmente con los primeros trabajos de Greenberg (1966), quien concede especial importancia a las secuencias lineares de las categorías gramaticales con funciones sintácticas, y establece, sobre fundamentos empíricos, unos universales ilimitados, implicacionales y estadísticos que plantean las posibles e imposibles variaciones existentes en las lenguas.

Algunas de las deficiencias que presentan los postulados de este modelo han sido suplidas en parte por otros planteamientos tales como los tres principios de jerarquía: el de gravedad, de inestabilidad y de movilidad, así como el principio de armonía entre categorías.

Las dos propuestas universalistas - la Gramática Universal y la investigación tipológica- se han desarrollado paralela y aisladamente. En un somero intento de comparación, advertimos que ambas propuestas se oponen claramente por una diferencia conceptual en sus planteamientos iniciales y son enfocadas con un objetivo deductivo la primera y heurístico la segunda. Ambas se distinguen, además, por el aspecto - intensivo vs. extensivo- de sus respectivos presupuestos teóricometodológicos al formular sus generalizaciones sobre diferentes niveles: la GU lo hace a nivel abstracto de estructura subyacente y la tendencia tipológica, a nivel de representación superficial.

Por el contrario, los dos modelos de investigación muestran algunas similitudes cuando proponen unos parámetros predictivos sobre el tipo de variación que puede o no existir entre las lenguas. Por otra parte, para cualquier teoría tipológica, todas las lenguas naturales deberían ser adecuadamente descriptivas y explicativas, criterios asumidos por la teoría chomskyana y asociados respectivamente a la capacidad generativa fuerte y débil de las lenguas.

Entre otras cosas, se puede reprochar a la escuela generativa su falta de evidencia interlinguística en su contribución universalista, por estar basada en una sola lengua —el inglés - . Por otra parte, los estudios tipológicos, por su inductivismo, no permiten abordar satisfactoriamente el problema de la capacidad de aprendizaje.

Sin embargo, los resultados de los estudios tipológicos pueden ser integrados en los que se obtienen a partir de la GU, gracias a la contribución de la tradición generativa con su modelo general para la fijación de parámetros, tales como los de la teoría de la marcación (teoría X barra, teoría «theta», etc.) que explica los 
mecanismos de la adquisición del lenguaje ${ }^{1}$. Esta integración podría, a su vez, revertir en una provechosa revisión de la teoría gramatical. Aunque adopta una postura conciliadora, Comrie (1981) considera más importante trabajar sobre una gran variedad de lenguas, por detectar en la propuesta chomskyana algunos puntos - -teóricos y prácticos- vulnerables: su hipótesis, por ser apriorística, no puede confirmarse eficazmente. Tampoco sus universales abstractos implican una afirmación empírica. Sin embargo, Comrie no rechaza el innatismo para explicar algunos universales lingüísticos y reconoce la necesidad de establecer un continuo entre enunciacios abstractos y concretos.

Pese a sus diferencias conceptuales y metodológicas, es evidente que las dos propuestas universalistas podrían y deberían buscar un punto de encuentro para sus distintos criterios, máxime cuando uno de sus objetivos comunes va dirigido hacia el complejo problema de la adquisición de una segunda lengua (ASL) (vid. Abraham, 1989).

2.2: Existe actualmente una abundante literatura sobre el proceso de ASL. Éste ha sido tratado desde diferentes perspectivas disciplinares. Una de ellas - la universalista - pertenece a la teoría gramatical y está siendo objeto de considerables estudios desde hace unos años.

Las dos corrientes - GU y tipología— ofrecen un posible marco teórico para el estudio de la ASL y el análisis de las interlenguas (IL) ${ }^{2}$. Se podría formular unas hipótesis de trabajo en términos absolutos o parciales, es decir, se podría plantear que todos, ninguno o algunos principios universales válidos para lasL1 lo serían también para las IL. La primera hipótesis no implica que la gramática de la Ll y de la IL sean idénticas. Ambos sistemas, aunque presentan similitudes funcionales, se diferencian, obviamente, por la dimensión temporal y la situación lingüístico-cognitiva en que son adquiridos. Inversamente, a partir de estas diferencias existentes, se puede proponer la segunda hipótesis. Sin embargo, algunos estudios empíricos ${ }^{3}$ abogan por unos universales comunes a los dos sistemas, lo que permite desechar esta hipótesis. La postura intermedia no parece ofrecer gran interés ya que, si se presupone que sólo algunos universales de las L1 sirven para las IL, habría que pronosticar cuáles son estos principios comunes y cuáles no lo son.

\footnotetext{
' Vid. Williams (1981) para una aplicación alternativa de la teoría de la marcación a la estructura de la locución en el problema de la adquisición del lenguaje. Por lo que se refiere a la adquisición de una segunda lengua, White (1989) ha tratado de elaborar una teoría a partir de una configuración paramétrica de la GU. Por su parte, Rutherford (1988) ofrece una visión general sobre estudios universalistas de diferentes tendencias, los cuales han tratado también la adquisición de una $\mathbf{L} 2$ en una perspectiva paramétrica, aunque sugiere que la noción misma de «parámetro» necesita ser revisada clara y minuciosamente.

${ }^{2}$ Este término se aplica, generalmente, a las segundas lenguas adquiridas por adultos y ha sido acuñado por Selinker (1972). Sin embargo, su uso puede ser equívoco y dar lugar a una identificación con un sistema amalgamado. Entendemos que la llamada «interlengua» es en realidad un continuo interlingüístico donde se sitúan una serie de variedades desde L1 hasta L2. (vid infra 3.3.). Para Corder (1971), estas variedades serían un tipo de «dialecto idiosincrásico» y recibirían el nombre de «dialecto transitorio».

${ }^{3}$ Vid. por ej. los estudios de Schmidt sobre la sintaxis de algunas construcciones combinadas y los de Tarone sobre la estructura silábica, ambos citados en Eckman (1988).
} 
Por lo tanto, sería coherente adoptar la primera hipótesis, según la cual todos los universales aplicables a las L1 lo son también a las IL hasta obtener evidencia contraria.

Ahora bien, la teoría de la GU sostiene que los dos sistemas - L1 e IL-son semejantes, en lo que respecta a los universales, tanto a nivel de representación superficial como a un nivel más abstracto. En cambio, la propuesta de los tipologistas admite la posibilidad de idénticos universales sólo para el nivel superficial. Ambas posturas requieren una confirmación basada en una mayor evidencia empírica. Por lo que se refiere a los procesos de adquisición de una segunda lengua y de la lengua materna en relación con la GU, el estudio del desarrollo sintáctico en estos procesos ha dado lugar a posturas contradictorias. Las posibilidades de acceso a la GU por parte de los nativos y de los adultos que adquieren una $L 2$ no son equivalentes, y habría que distinguir entre los principios y los parámetros de la GU, por una parte, y las específicas propriedades linguísticas de la L2, por otra (vid. Andersen, 1989; White, 1989).

Por nuestra parte, si tuviéramos que optar por una u otra propuesta universalista, el modelo de la GU nos parece, a priori, más sostenible no tanto por su método deductivo como por el nivel de abstracción en que son formulados sus principios. Por otro lado, no pretendemos ahondar ni radicalizar aquí en cuestiones de lógica sobre teorías del método científico. Inductivismo y deductivismo tienen sus respectivos defensores y posiblemente la ciencia sólo avanza con la complementariedad de ambos métodos ${ }^{4}$.

\section{Sociolingüística y multilingüismo}

La sociolingüística comenzó a dar sus primeros pasos como disciplina independiente en la década de los sesenta - aunque el término «sociolinguistics» había sido utilizado por primera vez unos años antes ${ }^{5}$, a raíz de una conferencia celebrada en la Universidad de California (Los Angeles) en 1964. La publicación de esta conferencia (Bright, 1966) contenía diferentes contribuciones a la investigación sociolingüística sobre temas relacionados con la etnolingüística, la linguística antropológica y la dialectología social. También se organizó un seminario en la Universidad de Indiana (Bloomington) bajo la dirección de Ferguson, cuya obra (1959) significó una aportación interesante en los estudios de bilingüismo. Otra publicación de 1966, la del estudio pionero de Labov, sobre la estratificación social del inglés en New York, iba a dar un giro singular a la sociolinguística y a la investigación empírica sobre la variación lingüística.

\footnotetext{
${ }^{4}$ Para una discusión filosófica e histórica sobre estos métodos, vid. Popper (1985); Nickles (1990); Hodge \& Cantor (1990).

${ }_{5}^{5}$ El término se debe a H. C. Currie quien lo utilizó en un trabajo publicado en 1952: «A projection of socio-linguistics: The relationship of speech to social status», Southern Speech Journal, 18, pp: 2837 , motivado en parte por el entorno multilingüe y multicultural del estado de Tejas. Situaciones similares en zonas fronterizas de Estados Unidos con Méjico plantearon posteriormente la necesidad de aplicar unos principios sociolingüísticos al estudio de las hablas marginales, reforzada por el impacto que suponía el «U.S. Civil Rights Act» de 1964.
} 
A partir de entonces, la realidad social multilingüe y multicultural de Estados Unidos, Canada y otros países europeos favoreció el desarrollo de unas investigaciones que trataban la influencia del lenguaje sobre la sociedad y la cultura o viceversa. Surgieron así numerosos estudios, muchas veces calificados de sociolingüísticos, pero que no dejaban muy claramente establecidos sus objetivos y límites, y defendían más bien intereses de diversas disciplinas — sociología, etnografía, antropología- junto a intereses lingüísticos de diversa magnitud e índole.

Tal dispersión ha originado la necesidad de delimitar y clasificar varias disciplinas particulares para su posterior reagrupación en disciplinas más generales. Varios y diferentes han sido los intentos de realizar este reordenamiento, si bien la mayoría de ellos coinciden en distinguir una sociolingüística general o macrosociolingüística de una sociolingüística particular o microsociolingüística. En la primera, cabría incluir la sociología del lenguaje. La segunda agruparía la sociolingüística estricta y, de modo complementario, la etnografía de la comunicación (vid. Gimeno, 1990, pág. 133 ss.).

La sociología del lenguaje ha recibidoconsideraciones diversificadas ${ }^{6}$ con relación a la sociolingüística (estricta). A pesar de su proximidad a la sociología, se configura como una ciencia social interdisciplinaria interesada en la organización social del comportamiento lingüístico en una perspectiva comunitaria o intercomunitaria. Además, incluye el estudio de las actitudes lingüísticas (vid. Agheyisi y Fishman, 1970; Fishman, Cooper y Ma, 1971; Fishman, 1972).

En el marco de una sociolingüística menos general, la etnografía de la comunicación estaría conectada a la antropología cultural y representaría el componente pragmático de la sociolinguística estricta. Su unidad de análisis -la comunidad de habla - se define como un grupo de hablantes que comparten un conocimiento no sólo del uso lingüístico sino también del modo en que los acontecimientos comunicativos se organizan de acuerdo con unas determinadas reglas de la situación comunicativa. Cabe señalar la influencia ejercida por la etnometodología en la etnografía de la comunicación cuando ésta revisó a final de los años setenta sus principios teóricos y amplió su objeto de estudio a las normas sociales que regulan y estructuran el acto comunicativo, y particularmente, la conversación (vid. Gumperz y Hymes, 1972; Garfinkel, 1972; Gumperz, 1982; Stubbs, 1983; SavilleTroike, 1986).

3.1. Un objeto distinto y evidente es el de la sociolingüística estricta si asumimos que ésta es una disciplina eminentemente lingüística, cuyas señas de identidad no pueden hoy ser cuestionadas, especialmente entre los que se dedican a estudiar científicamente la estructura de la lengua en su contexto social. Podríamos decir que la sociolingüística, en este sentido, se ha constituido como una extensión del modelo abstracto de la reciente teoría linguística. De este modo, puede responder a cuestiones no resueltas por dicha teoría, tales como la descripción y explicación de la variación en la actuación sociolingüística de los hablantes en el seno de una

'Vid. Silva-Corvalán, 1988, pp: 4 y ss; López Morales, 1989, pp: 25 y ss. 
comunidad de habla, y la representación de esta variación en el último modelo gramatical generativo-transformacional.

Esta sociolingüística estricta se afianza y desarrolla con los trabajos de Labov $(1966 ; 1972)$ en los que se distinguen dos etapas: una primera, caracterizada por una concepción estructural y taxonómica de la lengua; otra posterior, de más trascendencia, enmarcada en los principios de la lingüística generativa, pero con sustanciosas aportaciones que han permitido legitimar el encuadre teórico de la nueva disciplina.

En este marco, ciertas consideraciones deben ser destacadas. En primer lugar, la variación observada en el habla ya no puede ser considerada fortuita y caprichosa. La actuación lingüística es sistemática y dependiente de unos factores sociales correlacionados con la recurrencia de ciertos elementos de la estructura lingüística. Las primeras investigaciones de Labov (1966) así lo confirman. La variación, pues, al ser inherente al sistema, queda incorporada a la descripción lingüística. Por otra parte, en cuanto al conjunto de reglas que configuran la competencia sociolingüística de la comunidad de habla, el concepto de opcionalidad queda sustituido por el de variabilidad.

3.2. La descripción y análisis de la variación lingüística pasa por la delimitación de una unidad estructural - la variable lingüistica - para su posterior estudio cuantitativo. Esta unidad integra «un conjunto de equivalencia de realizaciones o expresiones patentes de un mismo elemento o principio subyacente» (Cedergren, 1983, pág. 150). Dicho conjunto de realizaciones o variantes está definido por unos factores lingüísticos y sociales, y representado formalmente por una regla variable (vid. Labov, 1969).

Es preciso indicar que la variable lingüística es un instrumento heurístico y no representa en sí una teoría del lenguaje, pero ha contribuido a la revisión de algunos principios teóricos. El concepto de variable linguística ha suscitado alguna controversia por la restricción que impone a la representatividad semántica frente a la extensión del significado reclamada por la lingüística formal. Esto implica ciertas limitaciones cuando el análisis de la variación se sitúa en un nivel distinto del fonológico - - p. ej., el sintáctico- en cuyo caso el conceptó de significado referencial habría de ser ampliado al de equivalencia funcional a la hora de elaborar el modelo de análisis (vid. Labov, 1978; Lavandera, 1978). Para Sankoff (1988), el estudio estadístico de la variación se incluye en un paradigma descriptivointerpretativo e intenta explicar la estructura gramatical en el discurso y la polivalencia de las relaciones entre la forma y la función lingüísticas. Este último problema, en el caso de la variación sintáctica, se resuelve por el mecanismo de la neutralización en el discurso.

A través de un procedimiento matemático materializado en el programa VARBRUL por Rousseau y Sankoff (1978), el último modelo de regla variable incorpora el cálculo de probabilidad logarítmica, y convierte las frecuencias reales de las variantes observadas en un índice probabilístico de aplicación, para cada uno de los factores - lingüísticos y sociales- que componen la regla variable. Se contribuye así a la creación de un nuevo modelo de competencia sociolinguística. 
Los paradigmas matemático-estadísticos — tanto el de regla variable como el del análisis de componentes principales u otros- diseñados para el análisis de la variación han sido y siguen siendo aplicados con creciente entusiasmo fuera de nuestras fronteras (Poplack, 1979; López Morales, 1983a; Horvath, 1985, entre otros muchos) y en nuestro país, aunque más tímidamente (Gimeno, 1983; Montoya, 1986; Alturo y Turell, 1990; Samper, 1990). Sin embargo, no todos los lingüistas admiten la incorporación de la probabilidad en sus teorías. Este rechazo se debe principalmente a tres motivos: 1) la influencia ejercida por el nodelo generativo chomskyano; 2) una investigación lingüística que aboga por la individualidad y el análisis discreto para formular sus juicios sobre gramaticalidad, y 3 ) unos modelos lingüísticos algebraicos que explican la estructura subyacente de la lengua y que no admiten los modelos probabilísticos estándar (cfr. López Morales, 1983b).

Con todo, el análisis estadístico, si presenta ciertas ventajas, en parte por los medios técnicos que permite utilizar, tiene también sus limitaciones y dificultades. La cuestión importante es la correcta elección del modelo estadístico más adecuado a la investigación emprendida. Para esta elección se ha de tener en cuenta, principalmente, el marco teórico de la investigación y sus objetivos, el diseño de la muestra y las variables linguísticas y sociales que se pretende analizar. Existen diversas evidencias empíricas de utilización de paradigmas estadísticos alternativos al de la regla variable, los cuales incluyen una visión crítica de la cuantificación de los datos lingüísticos y sociales, y de su tratamiento matemático (vid. Romaine, 1982; McEntegart y Le Page, 1982; Horvath, 1985; Milroy, 1987, pág. 177 ss.). Por otra parte, desde un punto de vista teórico, metodológico y bibliográfico, contamos ya en nuestro país, afortunadamente, con recientes publicaciones que ofrecen una excelente y estimulante muestra de la creciente importancia concedida hoy a la sociolinguística, y que deberían despertar el interés de nuestros investigadores (vid. López Morales, 1983a; Moreno Fernández, 1988; Silva-Corvalán, 1988; López Morales, 1989; Gimeno, 1990).

3.3. El análisis del comportamiento bilingüe tiene un punto de partida válido en el estudio contrastivo de los sistemas en contacto, dentro de una perspectiva estructuralista. En ella, el concepto de interferencia lingüistica cuenta con una larga tradición bibliográfica iniciada por Weinreich (1953) y con diversas interpretaciones para los diferentes problemas de lenguas en contacto, las cuales no siempre resultaron socialmente satisfactorias. Desde una perspectiva sociolingüística amplia, los estudios sobre bilingüismo han sido abordados bajo diferentes tendencias: lenguas en contacto, basada en las medidas de interferencia, y diglosia y conflicto lingüístico, que estudia el comportamiento individual en función de las normas sociales de la comunidad de habla. Desde una sociolingüística estricta, el bilingüismo ha sido estudiado en la dirección del variacionismo.

Por lo que se refiere al funcionamiento lingüístico en la mente del hablante bilingüe, las opiniones son actualmente bastante coincidentes. Si bien en la actuación bilingüe los sistemas en contacto pueden parecer separados, en el nivel más abstracto de la competencia comunicativa, lo que posiblemente existe es un solo 
sistema complejo (Lx) constituido por elementos de L1 y L2 procedentes de todos los niveles de la lengua, pero con sus propias normas de uso (vid. Oksaar, 1983; Cummins, 1984).

Por otra parte, en virtud del isomorfismo que puede existir entre los procesos mentales y el modelo formal de la gramática, y basados en el modelo amalgamado de las lenguas criollas, algunos variacionistas (Bailey, 1973; Bickerton, 1973; Berdan, 1977) han propuesto un modelo de gramática denominada polilectal que incluye varios lectos de una lengua histórica particular, y que puede ser aplicado a diferentes casos de variación. Uno de ellos, la adquisición del lenguaje, puede ser explicado por la constante revisión que el niño realiza durante el proceso de aprendizaje para acercarse asintóticamente a esta gramática polilectal. Asimismo, en cualquier comunidad de habla donde no exista un bilingüismo claramente definido, como p. ej., el proceso de adquisición de una segunda lengua, las variedades linguísticas producidas constituyen unas gramáticas de transición situadas a lo largo de un continuo cuyos extremos estarían ocupados por las variedades más alejadas entre sí (cfr. Heidelberg Forschungsprojekt «PidginDeutsch», 1978). Un ejemplo de ello estaría en las comunidades alicantinas que disponen de un solo repertorio lingüístico. En ellas los lectos valenciano, valencianoalicantino, murciano-alicantino y murciano responden a gramáticas de transición a lo largo del continuo geográfico y social catalano-castellano (cfr. Gimeno, 1986).

Sin embargo, en otras comunidades multilingües, la variación lingüística puede traducirse por una gramática de cambio de código resultante de la combinación de dos gramáticas monolingües en contacto. Este tipo de gramática genera en la estructura superficial un discurso regido por un conjunto de reglas sintácticas ordenadas jerárquicamente (vid. Sankoff y Poplack, 1981; Morales, 1986).

En general, podemos decir que la covariación de dos o más (sub)sistemas en las comunidades de habla que disponen de un único repertorio lingüístico puede conducir a diferentes soluciones: a) interferencia y cambio de código, b) amalgama o criollización, y c) sustitución de una lengua o variedad (A) por otra (B), o mantenimiento de ambas. El cambio lingüístico en curso sería un caso particular de interferencia y cambio de código.

\section{La adquisición de una segunda lengua desde una perspectiva sociolingüística}

Las disciplinas que han servido de base para un intento de construir una teoría de la ASL son varias: psicología, lingüística, sociología, etc. Además, el hecho de que el lenguaje sea una actividad humana estrechamente relacionada, por una parte con el pensamiento, por otra con la sociedad, ha favorecido la implicación de otras dos disciplinas recientes que reflejan esta doble conexión: la psicolingüística y la sociolingüística.

La noción de psicolingüística surge a principios de siglo del recíproco interés manifestado por psicólogos y lingüistas para acercar sus áreas de investigación, los primeros para comprender mejor la psique humana, los segundos para hallar una 
explicación de los cambios lingüísticos, sobre todo en una perspectiva histórica. Pero la fusión de ambas ciencias se vio realmente favorecida, por una parte, con la aportación de la lingüística saussureana a través de su orientación descriptiva y sincrónica, y por otra, con las tendencias que oponían la psicología europea a la americana: mentalismo y behaviorismo, respectivamente.

La psicolingüística quedó constituida como «ciencia de frontera» en un simposio celebrado en 1953 en la Universidad de Indiana, cuyas actas fueron publicadas el año siguiente por el psicólogo y antropólogo lingüista Osgood y Sebeok (cfr. Titone, 1979). Uno de los objetos de estudio de esta nueva disciplina es el desarrollo lingüístico en el niño, y por extensión, el aprendizaje y adquisición de una segunda lengua. Así lo vienen reflejando, hasta hace pocos años, un gran número de trabajos dedicados a ambos procesos.

4.1. No nos extenderemos sobre las diferentes teorías elaboradas desde esta perspectiva psicolingüística, pero sí conviene plantear aquí, aunque de un modo breve, algunas cuestiones. Por una parte, la clarificación de las nociones de aprendizaje y adquisición; por otra, la consideración de estos dos procesos referidos a la lengua materna y a una segunda lengua. Pero también interesa una puntualización sobre el objeto de la investigación.

En la literatura dedicada a la ASL no es frecuente encontrar unificación de criterios para la elaboración de un determinado marco teórico. Por otro lado, los abundantes estudios empíricos realizados tampoco ofrecen una homogeneidad en sus resultados. La cuestión más importante creemos que está en construir una buena teoría y aplicarla con una metodología adecuada, pero teoría y metodología están condicionadas a la previa delimitación del objeto de la investigación. Y sobre este particular, distinguimos dos principales centros de interés: el proceso y el producto de la ASL. El primero, por su carácter dinámico requiere un enfoque histórico; el estudio del segundo ha de situarse en el eje sincrónico. Uno supone analizar la evolución de las variedades inter lingüísticas en el tiempo, el otro implica describir la estructura de estas variedades.

En cuanto al aprendizaje y a la adquisición de una segunda lengua, podemos decir que son procesos, en parte naturales, determinados en su desarrollo por diversos factores (psicológicos, lingüísticos y sociales, etc.). Ambos están sometidos a ciertas reglas y en algunos casos pueden superponerse, pero el resultado final del proceso de aprendizaje no se obtiene sin la culminación del proceso de adquisición. Sin embargo, éste puede finalizar sin la influencia de aquél.

Krashen (1982, pág. 10 ss.) considera ambos procesos como distintos e independientes, capaces de desarrollar la competencia en una segunda lengua. Mientras que la adquisición es un proceso subconsciente, el aprendizaje es consciente y se caracteriza por la presencia de corrección de errores y la introducción de reglas aisladas en contextos lingüísticos artificiales. Según Krashen, el proceso de adquisición parece ser el más decisivo en el desarrollo completo de la competencia.

Si nos referimos a ambos procesos relacionados con una primera y una segunda lengua, también existen ciertas diferencias. El niño que adquiere su lengua materna 
establece relaciones directas entre ésta y la realidad, mientras que el individuo que aprende y/o adquiere una segunda lengua relaciona en primer lugar ésta con la interpretación conceptual que le proporciona su lengua materna, y sólo en un periodo posterior, establece relaciones entre la segunda lengua y la realidad.

4.2. Mientras que la ASL se sigue estudiando desde la perspectiva psicolingüística-teórica o aplicada-, recientemente, en el panorama de la investigación en segundas lenguas, ha aparecido un nuevo marco de referencia: el de la sociolinguística y el de la teoría lingüística general, con sus diferentes posibilidades de análisis: variación, pragmática, universales lingüísticos, análisis del discurso, etc. Son ya numerosos los estudiosos que se están aventurando en esta nueva senda de no fácil recorrido (vid. Flynn y O'Neil, 1988; Gass et al., 1989, Preston, 1989).

Una ojeada a las diferentes teorías sobre la ASL elaboradas durante las dos últimas décadas, basadas en la variación lingüística, permite entrever algunas posibilidades de investigación y unos nuevos puntos de partida para el análisis de las variedades interlinguísticas, en sus dos aspectos: el evolutivo y el estructural o sincrónico, es decir el análisis del sistema considerado ya sea como proceso, ya como producto.

Los nuevos modelos de ASL rechazaron en un principio la hipótesis contrastiva y la noción de interferecia, y se enmarcaron en una perspectiva sociolingüística. Sin embargo, no todos los investigadores aceptaron la sistematicidad de la variación. Otros, en cambio, aplicaron la noción de regla variable y establecieron un paralelismo entre el desarrollo de las variedades interlingüísticas y el paradigma dinámico de Bickerton y Bailey para caracterizar el cambio lingüístico. Es en la década de los ochenta cuando se inicia el análisis cuantitativo de la variación con la aplicación del programa VARBRUL 2 (cfr. Preston, 1989, pp: 239 ss.; vid. Tarone, 1983; 1988).

Por nuestra parte, nuestro interés en la investigación sobre la ASL se dirige especialmente hacia el análisis de la variación y su análisis cuantitativo. En el apartado 3.3. aludíamos brevemente al proceso que supone la adquisición de una segunda lengua y lo identificábamos con un caso de bilingüismo no claramente definido con posibilidad de análisis bajo la hipótesis variacionista de una gramática polilectal.

Cualquier investigación en el área de la adquisición de una segunda lengua debe partir de una exacta definición de su objeto de estudio con el fin de plantear claramente una hipótesis de trabajo. Sin embargo, no resulta fácil delimitar los procesos de adquisición y de aprendizaje debido a los diferentes tipos de variables -contextuales, sociales, psicológicas- que influyen en ellos. Incluso puede ocurrir que ambos procesos se superpongan cuando, p. ej., la adquisición de la segunda lengua se realiza en un contexto natural y es reforzada por una situación de aprendizaje en un contexto formal, a través de la educación escolar. Éste era el caso de una comunidad de adolescentes de origen francés que cursaban sus estudios en el Lycée Français de Alicante, y que tenían en su programa la asignatura de «Lengua española». Intentamos acercarnos a estos adolescentes para evidenciar una hipótesis de trabajo basada en la teoría variacionista laboviana. 


\section{Variación fonológica: metodología e hipótesis de trabajo}

El estudio que llevamos a cabo en el Lycée Français correspondía a nuestro deseo de contribuir modestamente a la investigación sobre ASL con la aplicación de una teoría sociolingüística al análisis de una variable fonológica, ya que a raíz de una preencuesta, habíamos observado hechos de variación en la actuación comunicativa del sistema español de algunos adolescentes francófonos.

Planteamos entonces una hipótesis de trabajo. Estos adolescentes formaban una comunidad de habla çon un único repertorio linguístico incluido en un continuo sociolectal que representaba su proceso de adquisición del español. En este continuo quedaban distribuidas unas «variedades intermedias» comprendidas entre sus dos extremos ocupados por las dos variedades de tipo estándar en covariación, la lengua fuente o nativa - francés - y la lengua objeto - español-.

Para el análisis cuantitativo de una variable fonológica - (s) - , se aplicó el modelo de regla variable mediante el programa matemático VARBRUL 2S. Éste que integra unos factores lingüísticos y sociales - nos permitió llegar a la competencia sociolingüística de esta comunidad de habla y explicarla por un modelo de gramática polilectal.

5.1. Los trabajos de Labov (1966) nos sirvieron de base para nuestra metodología que incluyó las siguientes etapas: diseño de la muestra, recogida de datos por el procedimiento de encuesta y análisis cuantitativo de los datos.

En principio, una investigación sociológica requiere que una muestra sea representativa y exhaustiva. De acuerdo con estos requisitos y con unas coordenadas previamente establecidas para la selección de variables independientes, acotamos una población de 26 sujetos y seleccionamos una muestra aleatoria de 16 informantes, con lo cual la representatividad quedaba ampliamente cubierta $(53,84 \%)^{7}$. Examinadas las posibles variables extralingüísticas que se nos ofrecían: edad, sexo, grupo social $y$ años de permanencia en el contexto natural, tuvimos que descartar dos de ellas: el sexo, ya que la mayoría de los sujetos eran chicas, y el grupo social, pues la práctica totalidad de los informantes pertenecían a un mismo estrato -medio medioFinalmente, la muestra fue distribuida en cuatro grupos: dos para la edad — de 9 a 13 años y de 14 a 17 años-y dos para la permanencia —más y menos de 5 años-

5.2. La recogida de datos se llevó a cabo mediante encuesta, con la grabación de cinco registros: dos orales familiar (A) y formal (B)-, y tres de lectura ${ }^{8}$ - texto (C), lista de palabras (D) y pares mínimos (E) - , diferenciados en función de la

${ }^{7}$ Según Labov (1966) los resultados de una investigación lingüística no quedan afectados cuando una muestra suficientemente estratificada se reduce al $0,025 \%$ del universo. Por otra parte, el bajo número de variables puede también justificar una pequeña muestra (López Morales, 1983a, pp: 25).

${ }^{8}$ Algunos sociolingüísticos se han resistido a incluir los registros orales y de lectura dentro de un mismo continuo, ya que, además de otras razones, la lectura no es un registro oral (vid. Romaine, 1982, pp: 118 y ss.; Terrel: «Dialectología» en López Morales (coord.): Introducción a la lingüística actual, Madrid, Playor, 1983, pp: 139, n. 13). 
formalidad del discurso. Las entrevistas se realizaron durante el mes de junio de 1983 y se prosiguieron durante los meses de abril y junio de 1984. El estilo oral más formal se obtuvo mediante la grabación de una conversación semidirigida de unos treinta minutos de duración. A continuación, fueron conseguidos los tres registros de lectura. Finalmente, cuando ya teníamos asegurado cierto grado de confianza con los alumnos, obtuvimos unos quince minutos de grabación de un registro familiar, con un magnetófono disimulado, después de agrupar a los sujetos en función de las dos variables independientes y solicitarles que hablaran de varios temas de su elección?.

Durante la preencuesta habíamos percibido en algunos sujetos cierta actitud favorable hacia la lengua española mientras que otros demostraban espontáneamente una menor motivación por el entorno natural y una mayor adhesión a su comunidad de origen, tanto en el aspecto lingüístico como en el sociocultural. Esto nos llevó a recoger, de un modo subjetivo, en las entrevistas personales posteriores, las posibles actitudes lingüísticas de estos adolescentes hacia el español. Una encuesta directa o indirecta nos hubiera permitido obtener una más clara correlación con los resultados definitivos del análisis sociolingüístico que la percibida subjetivamente. Sin embargo, esta encuesta complementaria no se pudo llevar a cabo por razones de tiempo, entre otras. En los estudios variacionistas que, desde una perspectiva inter disciplinaria, se propongan la explicación de una competencia comunicativa, un estudio de actitudes lingüísticas puede constituir un componente significativo. Pero, de hecho, los trabajos empíricos en este sentido son aún insuficientes.

\section{La variable lingüística: descripción}

Un estudio comparativo de los dos (sub)sistemas en covariación nos ofrecía la posibilidad de analizar diversas variables fonológicas y sintácticas. Sin embargo, nuestro interés se centró en el nivel fonológico por varias razones: las variables fonológicas, comparadas con las sintácticas, ofrecen mayor variación y son más fáciles de cuantificar. Además, la variación fonológica no implica diferencias del significado referencial asociadas a las variantes.

Conviene recordar que los componentes de esta comunidad de habla de origen francés, según nuestra hipótesis, realizaban su proceso de adquisición del español mediante la reestructuración constante de su gramática a partir del conjunto de reglas de $\mathrm{L} 1$ y del inventario fonológico de este (sub)sistema. Toda nueva regla adquirida cuyos rasgos sean extraños a la gramática de L1 será incorporada en su forma más general, es decir con unos rasgos no marcados ${ }^{10}$.

" El procedimiento de encuesta que seguimos en este estudio requiere hoy un planteamiento muy diferente siguiendo unas técnicas con las que en su día no pudimos contar (vid. Labov, 1984), especialmente para la obtención del registro familiar. En este caso, sabemos actualmente que las grabaciones llamadas «cándidas» realizadas con el micrófono disimulado no son aconsejables por dos razones, de ética una, de acústica la otra. No obstante, la «paradoja del observador» es un problema aún no resuelto del todo.

${ }^{10}$ Según el modelo variacionista de Bailey (1973), el cambio natural de una regla a otra sigue una dirección que va del rasgo más marcado o de la regla menos general al rasgo menos marcado o a la regla 
6.1. Aislados los segmentos y suprasegmentos del sistema español con rasgos ausentes en el inventario fonológico del francés, seleccionamos, de entre varios posibles, el segmento /s/ que podría implicar las siguientes variantes:

s1 [s] alófono fricativo predorso-dental sordo,

s2 $[\theta]$ alófono fricativo interdental sordo, y

s3 $[\mathrm{s} / \theta]$ alófono intermedio entre el fricativo predorso-dental y el interdental.

Al no existir la oposición $\theta / \mathrm{s}$ en su sistema primario, nuestros alumnos identificaban el fono fricativo interdental sordo $[\theta]$ del español con el fono fricativo predorso-dental tenso [s] de su sistema y producían un seseo predorso-dental. Nuestra hipótesis era que el grupo de más edad y el que contaba con una estancia inferior a 5 años darían una mayor frecuencia de la variante [s]. Para nuestro análisis, consideramos la variante predorsodental y la interdental -la variante intermedia presentó casos aislados-. Las dos variantes registradas, al no ofrecer grandes dificultades de percepción, no exigieron, a posteriori, el uso de un espectrógrafo.

Dentro del continuo estilístico, desde el registro menos formal al más formal, la variante no marcada [s] tendría una progresión decreciente, mientras que la variante marcada $[\theta]$ seguiría una progresión creciente. A modo de ejemplo, reproducimos el abanico estilístico de un sujeto (V.M.) de 12 años, con una permanencia de 8 años en España, del cual no pudimos obtener un registro familiar (vid. tabla I).

TABLA I

Abanico estilístico de V.M. para la variante $[\theta]$

Porcentajes Frecuencias

\begin{tabular}{|c|c|c|c|c|c|c|}
\hline & A & $\mathrm{B}$ & $\mathrm{C}$ & $\mathrm{D}$ & $\mathrm{E}$ & \\
\hline $\begin{array}{c}\text { Variante } \\
\text { s2 }\end{array}$ & - & 83 & 100 & 90 & 100 & -151998 \\
\hline
\end{tabular}

En este abanico vemos claramente una progresión creciente de la variante interdental que muestra una frecuencia de $83 \%$ en el registro oral no familiar (B) y un $100 \%$ en los registros de lectura de textos y de pares mínimos ${ }^{11}$.

más general. Para una teoría de la marcación, vid. Vennemann: «Cambio fonético y teoría de la marcación sobre la historia del sistema consonántico alemán», en Stockwell y Macaulay (eds.): Cambio lingüistico y teoría generativa, Madrid, Gredos, 1977, pp: 306-360; Guitart: «Fonología», en López Morales (coord.): Introducción a la lingüística actual, Madrid, 1983, pp: 83-113.

${ }^{11}$ La pequeña desviación observada en el registro de lectura de listas de palabras (contexto D) se explica por la ocurrencia de una variante predorso-dental en contexto fonológico implosivo (gaspacho [gaspaco]). 
6.2. Para obtener una variación más sistemática que la de un idiolecto, agrupamos los sujetos en función de las dos variables sociales, y el cómputo de las frecuencias de la variante interdental $[\theta]$ dio los porcentajes que aparecen en la tabla II para la variable años de permanencia.

El grupo «A», que representaba una estancia de más de cinco años, reflejaba unos valores superiores a los del grupo B, que reunía a los sujetos con menos de cinco años de permanencia. En ambos grupos las frecuencias seguían una progresión creciente salvo una ligera desviación en el registro de lectura de lista de palabras y de pares mínimos, explicable por la misma dificultad de estas pruebas.

\section{TABLA II}

Valores de $[\theta]$ en función de los años de estancia («A»: más de 5 años; «B»: menos de 5 años)

Grupos Porcentajes

$\begin{array}{lrllll} & \mathrm{A} & \mathrm{B} & \mathrm{C} & \mathrm{D} & \mathrm{E} \\ \text { «A»} & 86,2 & 89,4 & 90,7 & 89,7 & 96,8 \\ \text { «B» } & 6,7 & 45,1 & 53,3 & 66,6 & 56,2\end{array}$

En cuanto a los grupos 1 y 2 que representaban a los alumnos con edad comprendida entre los 9 y 13 años y entre los 14 y 17 años, respectivamente, las ocurrencias de la variante interdental dieron los porcentajes que aparecen en la tabla III siguiente:

TABLA III

Valores de $[\theta]$ en función de la edad

(Grupo 1: de 9 a 13 años; Grupo 2: de 14 a 17 años)

\begin{tabular}{llllll}
\hline Grupos & \multicolumn{5}{c}{ Porcentajes } \\
\hline & A & B & C & D & E \\
1 & 62,7 & 57,3 & 62,7 & 65,3 & 67,5 \\
2 & 26,8 & 64,4 & 87,7 & 100 & 91,6 \\
\hline
\end{tabular}

Para estos grupos, los valores también mostraban una progresión creciente exceptuando la desviación observada en el registro oral de mayor formalidad (B), para el grupo 1 de menor edad.

Con este análisis cuantitativo por frecuencias teníamos una primera confirmación parcial de nuestra hipótesis que fue reformulada. Una edad comprendida entre los 
14 y 17 años y una estancia superior a 5 años eran los factores que favorecían una mayor frecuencia de la variante interdental y que, por lo tanto, actuaban desfavorablemente en el fenómeno del seseo.

6.3. Desde un punto de vista descriptivo, el análisis cuantitativo de la variación reflejado en porcentajes puede ser válido pero insuficiente si queremos situarnos en el plano explicativo de la competencia sociolingüística. En este sentido, la inferencia estadística ofrece la posibilidad de inducir de los resultados obtenidos para unos cuantos elementos, cifras válidas para la población total a que estos elementos pertenecen. De este modo, permite el estudio del mundo real a partir de los esquemas teóricos de comportamiento del mundo ideal.

Los estudios variacionistas empíricos pueden beneficiarse hoy de esta aportación de la estadística matemática, gracias a los últimos avances teóricos. Éstos traducen las frecuencias a índices probabilísticos para cada uno de los rasgos lingüísticos y sociales que componen una regla variable (vid. 3.2.).

Consideradas las dos variantes de la variable (s), a la variante predorsodental asignamos los rasgos [+distribuido] y [+estridente] ausentes en la variante interdental, la cual sería definida como [+coronal] y $[+ \text { anterior }]^{12}$.

De acuerdo con estos rasgos, formulamos la siguiente regla variable:

$$
\left[\begin{array}{l}
+ \text { cons } \\
-\operatorname{voc}
\end{array}\right] \longrightarrow\left\langle\begin{array}{l}
+ \text { distr } \\
+ \text { estr }
\end{array}\right\rangle /\left(\begin{array}{l}
-- \\
+ \text { cor } \\
+ \text { ant }
\end{array}\right)
$$

la cual iría asociada a una cantidad específica $p$ que indicaría su probabilidad de aplicación. Una serie de factores independientes — variables extralingüísticasfavorecerían en mayor o menor medida la aplicación de la regla.

Dentro del continuo sociolectal que representaba el proceso de adquisición del español de estos adolescentes, habíamos optado por considerar la direccionalidad del cambio lingüístico hacia la variante marcada [s]. Reformulamos la hipótesis de que el grupo de más edad y con más de 5 años de estancia registraría una mayor probabilidad para la aplicación de la regla de interdentalización, es decir que los índices probabilísticos para estas variables independientes serían superiores a 0.5 .

En consecuencia, sometimos los datos sociolingüísticos al tratamiento estadísticos en un procesoinformático mediante el programa VARBRUL $2 S^{13}$.

12 Sobre la naturaleza de estos rasgos, vid. Otero: Evolución y revolución en romance. Mínima introducción a la fonología, 2 vols., Barcelona, Seix Barral, 1971/1976; Contreras y Lleó: Aproximación a la fonología generativa, Barcelona, Anagrama, 1982.

1.3 Agradecemos sinceramente la gentileza de los profesores H. López Morales y F. Gimeno Menéndez, los cuales hicieron posibles la obtención y aplicación de este programa. Hacemos extensiva nuestra gratitud al equipo del Centro de Proceso de Datos de la Universidad de Alicante por su valiosa ayuda técnica. 


\section{Análisis e interpretación de los datos}

Consideramos que la regla de interdentalización podría presentar una probabilidad de aplicación en función de dos grupos de restricciones variables mutuamente excluyentes:

1) la estancia: más de 5 años (factor $X)$ y menos de 5 años ( $Y$ ),

2) los registros contextuales: familiar (A), esmerado (B), lectura de textos (C), de lista de palabras (D) y de pares mínimos (E).

Después de combinar estos dos grupos de factores, fueron sometidas al programa las frecuencias observadas de la variante interdental sobre el número total de casos con posibilidad de aplicación de la regla. Sin embargo, después de unos primeros resultados, tuvimos que aislar el registro familiar, ya que aparecía un pequeño desajuste entre los casos observados y los valores pronosticados.

7.1. Las tablas IV y V muestran los nuevos resultados con valores más ajustados y unos índices de probabilidad situados en 0.75 para el factor $X$ (más de 5 años de estancia) que resultó reflejar el valor más significativo, y en 0.57 para el factor D (lista de palabras) que también justificaba una influencia favorable para la aplicación de la regla de interdentalización.

\section{TABLA IV}

Datos de la frecuencia observada de la variante $[\theta]$ para casos totales de la variable (s), excluido el factor $A$, con valores pronosticados entre paréntesis

\begin{tabular}{|c|c|c|c|c|}
\hline $\begin{array}{l}\text { Núm. de } \\
\text { aplicaciones }\end{array}$ & Total & $\begin{array}{l}\text { Grupo 1: } \\
\text { estancia }\end{array}$ & $\begin{array}{l}\text { Grupo 2: } \\
\text { reg.cont. }\end{array}$ & $\begin{array}{l}\text { chi } \\
\text { cuadrado }\end{array}$ \\
\hline $93(92.10)$ & 104 & $X$ & B & 0.076 \\
\hline $69(69.32)$ & 76 & $X$ & C & 0.018 \\
\hline $35(36.61)$ & 39 & $X$ & $\mathrm{D}$ & 1.158 \\
\hline $31(29.83)$ & 32 & $X$ & $\mathrm{E}$ & 0.676 \\
\hline $83(83.92)$ & 184 & $\mathrm{Y}$ & B & 0.019 \\
\hline $40(39.71)$ & 75 & $\mathrm{Y}$ & $\mathrm{C}$ & 0.004 \\
\hline $26(24.33)$ & 39 & Y & $\mathrm{D}$ & 0.301 \\
\hline $18(19.14)$ & 32 & $\mathrm{Y}$ & $\mathrm{E}$ & 0.170 \\
\hline 395 & 581 & & & 2.423 \\
\hline
\end{tabular}




\section{TABLA V}

Probabilidades de aplicación de la regla de interdentalización con coeficientes para cada factor, excluido el contexto $A$

\begin{tabular}{|c|c|c|c|}
\hline Po & 0.790 & & \\
\hline P est.(+5 a.) & 0.752 & P est. ( -5 a. $)$ & 0.248 \\
\hline P cont. B & 0.404 & P cont. C & 0.477 \\
\hline P cont. D & 0.573 & P cont. E & 0.546 \\
\hline
\end{tabular}

7.2. Otro grupo de restricciones que podían afectar a la aplicación de la regla de interdentalización era el formado por:

1) la edad: de 9 a 13 años (factor $V$ ) y de 14 a 17 años (Z),

2) los registros contextuales (A, B, C, D y E).

Los primeros resultados nos dieron nuevamente un pequeño desajuste a causa de una interacción de los datos correspondientes al registro familiar con la regla variable. Volvimos a aislar este registro y los resultados obtenidos son los que figuran en las tablas VI y VII.

\section{TABLA VI}

Datos de la frecuencia observada de la variante fonética $[\theta]$ para casos totales de la variable (s), excluido el factor $\mathbf{A}$

\begin{tabular}{lcccc}
\hline $\begin{array}{l}\text { Núm. de } \\
\text { aplicaciones }\end{array}$ & Total & $\begin{array}{c}\text { Grupo 1: } \\
\text { edad }\end{array}$ & $\begin{array}{c}\text { Grupo 2: } \\
\text { reg.cont. }\end{array}$ & $\begin{array}{l}\text { chi } \\
\text { cuadrado }\end{array}$ \\
\hline $78(69.36)$ & 136 & $\mathrm{~V}$ & $\mathrm{~B}$ & 2.196 \\
$59(62.35)$ & 94 & $\mathrm{~V}$ & $\mathrm{C}$ & 0.536 \\
$32(35.95)$ & 49 & $\mathrm{~V}$ & $\mathrm{D}$ & 1.631 \\
$27(28.56)$ & 40 & $\mathrm{~V}$ & $\mathrm{E}$ & 0.299 \\
$98(106.78)$ & 152 & $\mathrm{Z}$ & $\mathrm{B}$ & 2.431 \\
$50(46.58)$ & 57 & $\mathrm{Z}$ & $\mathrm{C}$ & 1.373 \\
$29(25.00)$ & 29 & $\mathrm{Z}$ & $\mathrm{D}$ & 4.638 \\
$22(20.40)$ & 24 & $\mathrm{Z}$ & $\mathrm{E}$ & 0.836 \\
\hline 395 & $\underline{581}$ & & & $\underline{13.941}$ \\
\hline
\end{tabular}


Estas interacciones de factores nos llevan a recordar que el programa de regla variable sólo es operativo con un análisis que previamente haya aislado los factores o grupos de factores susceptibles de interferir en el conjunto de las restricciones ${ }^{14}$. En nuestra investigación, hubiera sido necesario, pues, un nuevo análisis de estos factores. Por otra parte, el modelo de regla variable resulta más operativo con una gran cantidad de frecuencias y de variables independientes.

TABLA VII

\section{Probabilidades de aplicación de la regla de interdentalización con coeficientes para cada factor, excluido el contexto A}

\begin{tabular}{lccc}
\hline Po & 0.745 & & \\
\hline P edad (913) & 0.399 & P edad (14-17) & 0.601 \\
\hline P cont. B & 0.349 & P cont. C & 0.504 \\
\hline P cont. D & 0.587 & P cont. E & 0.563
\end{tabular}

Los resultados de la tabla VII confïmaban la aplicación de la regla de interdentalización para una edad comprendida entre los 14 y 17 años $(0.60)$ y en el contexto $\mathrm{D}(0.58)$. El registro de lectura de textos no tenía ningún efecto sobre la regla $(0.50)$. Para los dos grupos de restricciones analizados, el registro oral esmerado bloqueaba la regla variable.

\section{Conclusiones}

Este trabajo empírico nos permitió poner al descubierto, entre los alumnos francófonos del Lycée Français de Alicante, una comunidad de habla donde covariaban dos (sub)sistemas con un solo repertorio lingüístico. Con la aplicación de una teoría sociolingüística variacionista, habíamos accedido a la competencia polilectal de esta comunidad mediante la estructuración de la variable sociolingüística (s).

El tratamiento estadístico de los datos nos indicó que eran tres los factores que más favorecían la aplicación de la regla variable para la variante interdental $[\theta]$ :

a. una estancia de más de 5 años en España,

b. una edad comprendida entre los 14 y 17 años, y

c. un registro de lectura de listas de palabras.

${ }^{17}$ Vid. W. Labov y T. Labov: «L'apprentissage de la syntaxe des interrogations», Langue Fransaise, 34, 1977, pp: 52-80. 
Sin embargo, estos resultados nos obligaron a un replanteamiento de nuestra hipótesis inicial. A través del análisis de regla variable del segmento fonológico /s/, pudimos deducirque la variación sociolingüística de estos adolescentes franceses no presentaba la regularidad que habíamos esperado dentro del continuo estilístico, y reflejaba una ruptura entre los registros orales y los de lectura. Sin embargo, esta discontinuidad es explicable por cuanto ambos registros no son equiparables.

Por otra parte, no conviene olvidar los dos con textos en los que estos alumnos desarrollaban su proceso de adquisición del español. El contexto escolar, esencialmente formal, es el que contribuía al aprendizaje de los rasgos de prestigio de la lengua objeto. Pero el contexto natural, extraescolar, era el que favorecía el cambio natural, es decir, la producción de rasgos no marcados. A ello contribuían, por una parte, la interacción lingüística con los amigos y compañeros nativos, en la que el discurso requería menor atención, y por otra, el entorno familiar y el contacto con amigos franceses, en los que predominaba el sistema primario.

Es evidente que este contexto natural fue el que más influyó en la ocurrencia de la variante predorso-dental $[\mathrm{s}$ ] en nuestros estudiantes. La selección de más variables extralingüísticas (intensidad del contacto con hablantes españoles, actitudes lingüísticas, mayor estancia en España, mayor edad) nos hubiera sin duda permitido determinar con más precisión el factor más favorable a la aplicación de la regla variable $y$, en consecuencia, la direccionalidad del cambio. Por otra parte, la prolongación de su estancia y de sus estudios en España, podría favorecer el mantenimiento de los dos sistemas coordinados, siempre que exista, entre otros factores, un equilibrio entre una identificación con su comunidad lingüística de origen y una actitud favorable hacia la lengua y la cultura españolas.

En suma, podemos decir que este trabajo tenía una doble intencionalidad. Por una parte, pretendíamos dar una pequeña muestra, a nivel empírico de las posibilidades que ofrecen hoy los últimos avances sociolingüísticos en la cuantificación estadística de la variación, para acercarnos al aspecto explicativo de esta última. Por otra parte, a nivel teórico, queríamos esbozar un nuevo marco de referencia para investigaciones futuras en este complejo entramado que constituyen la adquisición y aprendizaje de las segundas lenguas y la Gramática Universal. Se ha empezado a caminar y no son escasas las dificultades, pero disponemos ya de algunos instrumentos para superarlas, si asumimos plenamente la necesidad de un marco interdisciplinario para esta interesante área de investigación.

\section{Referencias bibliográficas}

ABRAHAM, W. (1989): «The Chomskyan Approach vs. Greenberg's Typological Approach», en KEFER, M.y J. van der AUWERA (eds.), Universals of Language, Belgian Journal of Linguistics, 4, Bruxelles: Editions de l'Université de Bruxelles, pp: 9-25.

AGHEYISI, R. y J. A. FISHMAN (1970): «Language Attitudes Studies», Anthropological Linguistics, 12, pp: 137-157.

ALTURO, N. y M. T. TURELL (1990): «Language Attitudes Studies», 
Anthropological Linguistics, 12, pp: 137-157.

ANDERSEN, R. W. (1989): «La adquisición de la morfología verbal», Lingüística, 1, pp: $90-142$.

BAILEY, Ch. J. N. (1973): Variation and Linguistic Theory, Arlington: Center for Applied Linguistics.

BERDAN, R. (1977): «Polylectal Comprehension and Polylectal Grammar», en FASOLD, R. W.y R. W.SHUY (eds.), Studies in Language Variation. Semantics, Syntax, Phonology, Pragmatics, Social Situations, Ethnographic Approaches, Washington, D.C.: Georgetown University Press, pp: 12-29.

BICKERTON, D. (1973): «The structure of Polylectal Grammars», en SHUY, R. W. (ed.), Linguistics and Language Study (23rd Round Table Meeting). Sociolinguistics: Current Trends and Prospects, Washington, D.C.: Georgetown University Press, pp: 17-42.

BRIGHT, W. (ed.)(1966): Sociolinguistics Proceedings of the UCLA Sociolinguistics Conference, The Hague: Mouton.

CEDERGREN, H. (1983): «Sociolingüística», en LÓPEZ MORALES, H. (coord.), Introducción a la Lingüística actual, Madrid: Playor, pp: 147-165.

COMRIE, B. (1981): Universales del lenguaje y tipologíalingüística, Madrid, Gredos, 1988.

CORDER, S. P. (1971): «Idiosyncratic Dialects and Error Analysis», IRAL, vol. IX (2), pp: 147-160. Trad. fr. «Dialectes idiosyncrasiques et analyse d'erreurs», Langages, 57, 1980, pp: 17-28.

CUMMINS, J. (1984): Bilingualism and Special Education: Issues in Assessment and Pedagogy, Clevedon: Multilingual Matters.

CHOMSKY, N. (1965): Aspects of the Theory of Syntax, Cambridge. Trad. esp. Aspectos de la teoría de la sintaxis, Aguilar: Cultura e Historia, 1970.

CHOMSKY, N. (1971): El lenguaje y el entendimiento, Barcelona: Seix Barral, 1980.

ECKMAN, F. R. (1988): «Typological and parametric views of Universals in Second Language Acquisition», en FLYNN, S. y W. O'NEIL (eds.), Linguistic Theory in Second Language Acquisition, Dordrecht: Kluwer Academic Publishers, pp: 417-429.

FERGUSON, Ch. A. (1959): «Diglosia», Word, 15, pp: 325-340.

FISHMAN, J.A. (1968): «Sociolinguistic perspective on the study of bilingualism», en FISHMAN, J. A., R. L. COOPER, R. MA et al., Bilingualism in the Barrio, Bloomington: Indiana University, $2^{2}$ ed. rev., 1975, pp: 557-582.

FISHMAN, J. A. (1972): The sociology of language, Rowley: Newbury House Publishers. Trad. esp. Sociología del lenguaje, Madrid: Cátedra, 1979.

FISHMAN, J. A., R. L. COOPER y R. MA et al. (1971): Bilingualism in the Barrio, $2^{a}$ ed. rev., Bloomington: Indiana University Publications, 1975.

FLYNN, S. y W. O'NEIL (eds.) (1988): Linguistic Theory in Second Language Acquisition, Dordrecht: Kluwer Academic Publishers.

GARFINKEL, H. (1972): «Remarks on ethnomethodology», en GUMPERZ, J. J. y HYMES, D. (eds.), Directions in sociolinguistics: The ethnography of communication, New York: Holt, Rinehart and Winston, pp: 301-324. 
GASS, S., C. MADDEN, D. PRESTON y L. SELINKER (eds.) (1989): Variation in Second Language Acquisition, Vol I y II, Clevedon: Multilingual Matters.

GIMENO, F. (1983): «De sociolinguíistica histórica: Tradición grafemática y variable fonológica», Boletín de la Academia Puertorriqueña de la Lengua española, San Juan de P. Rico, pp: 71-86.

GIMENO, F. (1986): «Sustitución linguíística en las comunidades de habla alicantinas», ELUA, 3, pp: 237-267.

GIMENO, F. (1990): Dialectología y sociolingüística españolas, Alicante, Universidad de Alicante.

GIMENO, F. y B. MONTOYA (1989): Sociolingüíca, Valencia: Servei de Publicacions de la Universitat de València.

GREENBERG, J.H. (1966): «Some universals of grammar with particular reference to the order of meaningful elements", en GREENBERG, J. H. (ed.), Universals of Language, Cambridge, Mass.: MIT Press; reimp. en Keiler, 1972, pp: 306337.

GUMPERZ, J. J. (1982): Discourse strategies, Cambridge: Cambridge University Press.

GUMPERZ, J. J. y D. HYMES (eds.) (1972): Directions in Sociolinguistics: The Ethnography of Communication, New York: Holt, Rinehart and Winston.

HEIDELBERGER FORSCHUNGSPROJEKT «PIDGIN-DEUTSCH» (1978): «The Acquisition of German Syntax by Foreign Migrant Workers», en SANKOFF, D. (ed.), Linguistic Variation: Models and Methods, New York: Academic Press, pp: $1-22$.

HODGE, M. J. S. y N. CANTOR (1990): «The develpment of philosophy of Science since $1900 \%$, en OLBY, R. C. et al., Companion to the History of Modern Science, London: Routledge, pp: 838-852.

HORVATH, B. M. (1985): Variation in Australian English. The sociolects of Syntax, Cambridge, Cambridge University Press.

KEFER, M. y J. van der AUWERA (eds.) (1989): Universals of Language. Belgium Journal of Linguistics, 4, Bruxelles: Editions de l'Université de Bruxelles.

LABOV, W. (1966): The social stratification of English in New York City, Washington, D.C.: Center for Applied Linguistics, 1982.

LABOV, W. (1969): «Contraction, Delection and Inherent Variability of the English Copular», Rev. en Language in the Inner city. Studies in the Black English Vernacular, Oxford, Blackwell, 1977, pp: 65-129.

LABOV, W. (1972): Sociolinguistic Patterns, 1" ed., Oxford: Blackwell, 1978. Trad. fr. Sociolinguistique, Paris: Les Editions de Minuit, 1976.

LABOV, W. (1978): «Where does the sociolinguistic variable stop? A response to B. Lavandera», Texas Working Papers in Sociolinguistics, 44, Austin: Southwest Educational Development Laboratory.

LABOV, W. (1984): «Field methods of the project on linguistic change and variation» en BAUGH, J. y J. SHERZER (eds.), Language in Use, Englewood Cliffs: PrenticeHall, pp: 28-53.

LAVANDERA, B. (1978): «Where does the sociolinguistic variable stop?», Language in Society, 7, pp: 171-182. 
LÓPEZ MORALES, H. (1983a): Estratificación social del Español de San Juan de P. Rico, Méjico: Universidad Nacional Autónoma.

LÓPEZ MORALES, H. (1983b): «Lingüística estadística», en LÓPEZ MORALES, H. (coord.), Introducción a la lingüística actual, Madrid: Playor, pp: 209-225. LÓPEZ MORALES, H. (1989): Sociolingüustica, Madrid: Gredos.

McENTEGART, D. y R. B. LE PAGE (1982): "An appraisal of the statistical techniques used in The Sociolinguistic Survey of Multilingual Communities», en ROMAINE, S. (ed.), Sociolinguistics Variation in Speech Communities, London: Arnold, pp: 105-124.

MILROY, L. (1987): Observing and Analysing Natural Language, Oxford: Blackwell.

MONTOYA ABAD, B. (1986): Variació $i$ desplaçament de llengües a Elda $i$ a Oriola durant l'edat moderna, Alicante: Institut d'Estudis «Juan Gil Albert».

MORALES, A. (1986): Gramáticas en contacto: Análisis sintácticos sobre el español de Puerto Rico, Madrid: Biblioteca de autores de Puerto Rico.

MORENO FERNÁNDEZ, F. (1988): Sociolingüística en Estados Unidos. (19751985). Guía bibliográfica crítica, Málaga: Ed. Librería Agora.

NICKLES, T. (1990): «Discovery», en OLBY, R. C. et al., Companion to the History of Modern Science, London: Routledge, pp: 148-165.

OLBY, R. C. et al. (1990): Companion to the History of Modern Science, London: Routledge.

OKSAAR, E. (1983): «Multilingüismo y multiculturalismo desde el punto de vista del lingüista», en HUSEN, T. y S. OPPER (eds.), Educación multicultural y multilingüe, Madrid: Marcea, 1984, pp: 33-59.

POPLACK, S. (1979): «Function and process in a variable phonology» (tesis doctoral inédita), Philadelphia: University of Pennsylvania.

POPPER, K. (1935): La lógica de la investigación científica, Madrid: Tecnos, 1985.

PREST ON, D.R.(1989): Sociolinguistics and SecondLanguage Acquisition, Oxford: Blackwell.

ROMAINE, S. (1982): Sociohistorical linguistics: its status and methodology, Cambridge: Cambridge University Press.

ROTAETXE, K. (1988): Sociolingüistica, Madrid: Editorial Síntesis.

ROUSSEAU,P.yD.SANKOFF (1978): «Advances in Variable Rule Methodology», in SANKOFF, D. (ed.), Linguistic Variation. Models and Methods, New York: Academic Press, pp: 5769.

RUTHERFORD, W (1988): «Grammatical Theory and L2 Acquisition: A brief overview», in FLYNN, S. y W. O'NEIL (eds.), Dordrecht, pp: 404-416.

SAMPER PADILLA, J. A. (1990): Estudio sociolingüístico del español de Las Palmas de Gran Canaria, Las Palmas: Pérez Galdos.

SANKOFF, D. (1988): «Sociolinguistics and syntactic variation», en NEWMEYER, F. (ed.), Linguistics: The Cambridge Survey, Cambridge: Cambridge University Press, pp: 140-161.

SANKOFF, D. y S. POPLACK (1981): «A formal Grammar for codes-witching», en Papers in Linguistics, 14, 2, pp: 3-46.

SAVILLE-TROIKE, M. (1982): The Ethnography of communication. An 
introduction, New York: Basil Blackwell, 1986.

SELINKER, L. (1972): «Interlanguage», International Review of Applied linguistics in Language Teaching, 10, pp: 209-231.

SILVA-CORVALÁN, C. (1988): Sociolingüística: Teoría y análisis, Madrid: Alhambra.

STUBBS, M. (1983): Discourse analysis. The Sociolinguistic Analysis of Natural Language, Oxford: Blackwell.

TARONE, E. (1983): «On the variability of interlanguage systems», Applied Linguistics, 4/2, pp: 143-163.

TARONE, E. (1988): Variation in interlanguage, London: Edward Anold.

TITONE, R. (1979): Psycholinguistique appliquée, Paris: Payot.

WEINREICH, U. (1953): Languages in contact. Findings and Problems, $6^{\circ}$ ed., The Hague-Paris: Mouton, 1968. Trad. esp. Lenguas en contacto. Descubrimientos y problemas, Caracas: Ediciones de la Biblioteca de la Universidad Central, 1974.

WHITE, L. (1989): Universal Grammar and Second Language Acquisition, Amsterdam: John Benjamins Publishing.

WILLIAMS, E. S. (1981): «Language Acquisition, Markedness and Phrase Structure», en TAVAKOLIAN, S. L., Language Acquisition and Linguistic Theory, Cambridge: MIT Press, pp: 8-34. 